\title{
An improved superconvergence error estimate for the LDG method *
}

\author{
Slimane Adjerid ${ }^{1}$, Nabil Chaabane \\ Department of Mathematics Virginia Tech, Blacksburg, VA 24061-0123, USA
}

\begin{abstract}
In this manuscript we present an error analysis for the local discontinuous Galerkin method for a model elliptic problem on Cartesian meshes when polynomials of degree at most $k$ and an appropriate approximation of the boundary condition are used. This special approximation allows us to achieve $k+1$ order of convergence for both the potential and its gradient in the $L^{2}$ norm. Here we improve on existing estimates for the solution gradient by a factor $\sqrt{h}$.
\end{abstract}

Key words: Finite element method; Discontinuous Galerkin; Superconvergence; Elliptic problems.

\section{$1 \quad 1$ Introduction}

2 The discontinuous Galerkin (DG) finite element method was first used to solve the neutron equation [17]. Cockburn and Shu [11,13] extended the method to solve first-order hyperbolic partial differential equations of conservation laws. They also developed the Local Discontinuous Galerkin (LDG) method for convection-diffusion problems [12]. DG methods have been developed for other partial differential equations the reader may consult $[10,14,15,16]$ and the references cited therein for a detailed discussion of the history of DG methods and their applications.

10 The success of the DG method is due to one or more of the following properties: (i) does not require continuity across element boundaries, (ii) is locally

‡ This research was partially supported by the National Science Foundation (Grant Number DMS1016313, DMS0809262).

Email addresses: adjerids@math.vt.edu, Tel:(540) 2315945 (Slimane

Adjerid), nabilch@vt.edu (Nabil Chaabane).

1 Corresponding author. 
conservative, (iii) is well suited to solve problems on locally refined meshes with hanging nodes, (iv) exhibits strong superconvergence that can be used to estimate the discretization error, (v) has a simple communication pattern between elements with a common face that makes it useful for parallel computation and (vi) it can handle problems with complex geometries to higher order.

The LDG finite element method for solving one-dimensional convection- diffusion partial differential equations was introduced by Cockburn and Shu [12] and is based on the work by Bassi and Rebay [4]. Castillo et al. [6] presented the first a priori error analysis for the LDG method applied to a model elliptic problem. They considered arbitrary meshes with hanging nodes and elements of various shapes and studied general numerical fluxes. They showed that, for smooth solutions, the $L^{2}$ errors in $u$ and $\nabla u$, respectively, are of order $k+1 / 2$ and $k$ when polynomials of total degree not exceeding $k$ are used. Later, Cockburn et al. [9] presented a superconvergence result for the LDG method for a model elliptic problem on Cartesian meshes. They identified a special numerical flux for which the $L^{2}$ norms of the solution gradient and the potential are of orders $k+1 / 2$ and $k+1$, respectively, when tensor product polynomials of degree at most $k$ are used.

An optimal minimal dissipation LDG (md-LDG) method for one-dimensional convection-diffusion problems was first investigated in [7]. Later, the method was extended to two-dimensions on triangular meshes in [8] where the authors proved $\mathcal{O}\left(h^{k+1}\right)$ and $\mathcal{O}\left(h^{k}\right) L^{2}$ convergence rates, respectively, for the potential and solution gradient using the complete $k$-degree polynomial space $P_{k}$.

Cockburn et al. [9], at the end of section 4.3, stated the following: "These experiments justify our contention that the optimal order of convergence in q can be reached if the boundary conditions are piecewise polynomials of degree $k$. Our theoretical analysis does not explain this phenomenon". Computational results in section 3 and [2], respectively, for general LDG methods and the minimial dissipation LDG method suggest that the LDG solution of elliptic problems obtained by interpolating Dirichlet boundary conditions at Radau points is $\mathcal{O}\left(h^{k+1}\right)$ superconvergent for both the solution gradient and potential. In this manuscript we prove that, by approximating Dirichlet boundary conditions with appropriate projections or interpolations, both the LDG solution and its gradient on Cartesian meshes are $\mathcal{O}\left(h^{k+1}\right)$ convergent. The proof is carried out for the md-LDG method and its extension to other LDG methods is discussed in the next section. 


\section{Superconvergence error analysis}

Consider the following second-order elliptic boundary-value problem

$$
\begin{aligned}
-\Delta u & =f, \quad \text { in } \Omega=[-1,1]^{2}, \\
u & =g_{D}, \quad \text { on } \partial \Omega_{D}, \\
\nabla u \cdot \mathbf{n} & =\mathbf{g}_{N} \cdot \mathbf{n}, \quad \text { on } \partial \Omega_{N},
\end{aligned}
$$

for some given functions $g_{D}$ and $\mathbf{g}_{N}$, where $\partial \Omega=\partial \Omega_{D} \cup \partial \Omega_{N}$, $\mathbf{n}$ is the unit outer normal vector on $\partial \Omega$ and the measure of $\partial \Omega_{D}$ is nonzero.

In our analysis, we select the boundary conditions and the source term $f(x, y)$ such that the exact solution, $u(x, y)$, is a smooth function. In order to construct the LDG formulation of Cockburn et al. [9], we first introduce an auxiliary variable $\mathbf{q}=\nabla u$ and transform the elliptic problem (2.1) to the following system of first-order differential equations

$$
\begin{aligned}
\mathbf{q} & =\nabla u, \quad \text { in } \Omega, \\
-\nabla \cdot \mathbf{q} & =f, \quad \text { in } \Omega, \\
u & =g_{D}, \quad \text { on } \partial \Omega_{D}, \\
\mathbf{q} \cdot \mathbf{n} & =\mathbf{g}_{N} \cdot \mathbf{n}, \quad \text { on } \partial \Omega_{N} .
\end{aligned}
$$

Next, we partition our domain $\Omega$ into a rectangular mesh $\mathscr{T}$ consisting of $N=n \times n$ elements $K_{i j}=\left[x_{i}, x_{i+1}\right] \times\left[y_{j}, y_{j+1}\right], i, j=0,1, \ldots, n-1$, where $x_{i}=y_{i}=-1+i h, i=0,1, \ldots, n$ and $h=2 / n$. Now we multiply (2.2a) and (2.2b) by arbitrary smooth test functions $v$ and $\mathbf{r}$, respectively, integrate over an arbitrary element $K$, and apply the divergence theorem to write

$$
\begin{aligned}
\int_{K} \mathbf{q} \cdot \mathbf{r} d x & =-\int_{K} u \nabla \cdot \mathbf{r} d x+\int_{\partial K} u \mathbf{r} \cdot \mathbf{n} d s \\
\int_{K} \mathbf{q} \cdot \nabla v d x & =\int_{K} f v d x+\int_{\partial K} v \mathbf{q} \cdot \mathbf{n} d s .
\end{aligned}
$$

Let $Q_{k}(K)$ denote the tensor product space on $K$ consisting of polynomials where the degree in each variable does not exceed $k$, and define

$$
\begin{aligned}
M_{N} & :=\left\{\mathbf{q} \in\left(L^{2}(\Omega)\right)^{2} \quad:\left.\quad \mathbf{q}\right|_{K} \in Q_{k}(K)^{2}\right\}, \\
V_{N} & :=\left\{u \in L^{2}(\Omega):\right.
\end{aligned}
$$

The LDG formulation consists of finding $\left(\mathbf{q}_{N}, u_{N}\right) \in M_{N} \times V_{N}$ that satisfies

$$
\begin{aligned}
\int_{K} \mathbf{q}_{N} \cdot \mathbf{r} d x & =-\int_{K} u_{N} \nabla \cdot \mathbf{r} d x+\int_{\partial K} \hat{u}_{N} \mathbf{r} \cdot \mathbf{n} d s \\
\int_{K} \mathbf{q}_{N} \cdot \nabla v d x & =\int_{K} f v d x+\int_{\partial K} v \hat{\mathbf{q}}_{N} \cdot \mathbf{n} d s
\end{aligned}
$$

for all $(\mathbf{r}, v) \in M_{N} \times V_{N}$ and for all $K \in \mathbf{T}$. Here $\mathbf{n}$ is the unit outward normal vector to the edges $\partial K$ and the numerical fluxes $\hat{\mathbf{q}}_{N}$ and $\hat{u}_{N}$ are the discrete 
approximations of the traces of $\mathbf{q}$ and $u$ on element boundaries. In order to complete the definition of the md-LDG method we need to select the fluxes $\hat{\mathbf{q}}_{N}$ and $\hat{u}_{N}$ on $\partial K$. Let the mean value $\{\cdot\}$ and jump $\llbracket \cdot \rrbracket$ of a scalar function $u_{N}$ and a vector $\mathbf{q}_{N}$ at $(x, y)$ on an edge of $\partial K$ be defined as

$$
\begin{aligned}
& \{u\}(x, y)=\frac{1}{2}\left(u^{+}(x, y)+u^{-}(x, y)\right), \quad\{\mathbf{q}\}(x, y)=\frac{1}{2}\left(\mathbf{q}^{+}(x, y)+\mathbf{q}^{-}(x, y)\right), \\
& \llbracket u \rrbracket(x, y)=\left(u^{+}(x, y)-u^{-}(x, y)\right) \mathbf{n}, \quad \llbracket \mathbf{q} \rrbracket(x, y)=\left(\mathbf{q}^{+}(x, y)-\mathbf{q}^{-}(x, y)\right) \cdot \mathbf{n},
\end{aligned}
$$

where $u^{+}$is the limit of the solution on $K$ and $u^{-}$is the limit of the solution of an adjacent element sharing $\partial K$, i.e., for $(x, y) \in \partial K$, and $\mathbf{n}$ is the outer unit normal on $\partial K$ we have

$$
u^{+}(x, y)=\lim _{\epsilon \rightarrow 0^{-}} u((x, y)+\epsilon \mathbf{n}), \quad u^{-}(x, y)=\lim _{\epsilon \rightarrow 0^{+}} u((x, y)+\epsilon \mathbf{n}) .
$$

We follow [9] to define the numerical fluxes $\hat{u}$ and $\hat{\mathbf{q}}$ on interior edges by introducing an auxiliary vector $\mathbf{v}$ and write

$$
\begin{aligned}
& \hat{\mathbf{q}}=\{\mathbf{q}\}-C_{11} \llbracket u \rrbracket-\mathbf{C}_{12} \llbracket \mathbf{q} \rrbracket, \\
& \hat{u}=\{u\}+\mathbf{C}_{12} \cdot \llbracket u \rrbracket .
\end{aligned}
$$

The parameters are defined on each edge of $\partial K$ such that $C_{11} \geq 0$ and $\mathbf{C}_{12}$ is given by

$$
\mathbf{C}_{12} \cdot \mathbf{n}=\frac{1}{2} \operatorname{sign}(\mathbf{v} \cdot \mathbf{n})
$$

We note that the vector $\mathbf{v}$ is an arbitrary but fixed vector with nonzero components as illustrated in Figure 2.1. Without loss of generality, from now on we assume that $\mathbf{v}$ has strictly positive components, thus, $\hat{u}$ on a horizontal edge is the limit of $u$ from below while on a vertical edge it is the limit of $u$ from the left. $\hat{\mathbf{q}}$ on a horizontal edge is the limit of $\mathbf{q}$ from above while $\hat{\mathbf{q}}$ on a vertical edge is the limit from the right. We also define

$$
\partial \Omega^{-}=\{(x, y) \in \partial \Omega \mid \mathbf{v} \cdot \mathbf{n}<0\}, \quad \partial \Omega^{+}=\{(x, y) \in \partial \Omega \mid \mathbf{v} \cdot \mathbf{n}>0\} .
$$

If $\partial \Omega_{D}^{ \pm}=\partial \Omega_{D} \cap \partial \Omega^{ \pm}$, we let $\mathscr{E}, \mathscr{E}_{D}, \mathscr{E}_{N}, \mathscr{E}^{ \pm}, \mathscr{E}_{D}^{ \pm}$respectively, denote the sets of all edges in $\partial \Omega, \partial \Omega_{D}, \partial \Omega_{N}, \partial \Omega^{ \pm}, \partial \Omega_{D}^{ \pm}$. Finally the set of all interior edges is defined as $\mathscr{E}_{I}=\mathscr{E} \backslash\left(\mathscr{E}_{D} \cup \mathscr{E}_{N}\right)$.

Letting $C_{11}$ be a positive constant we define the numerical flux on the boundary as 


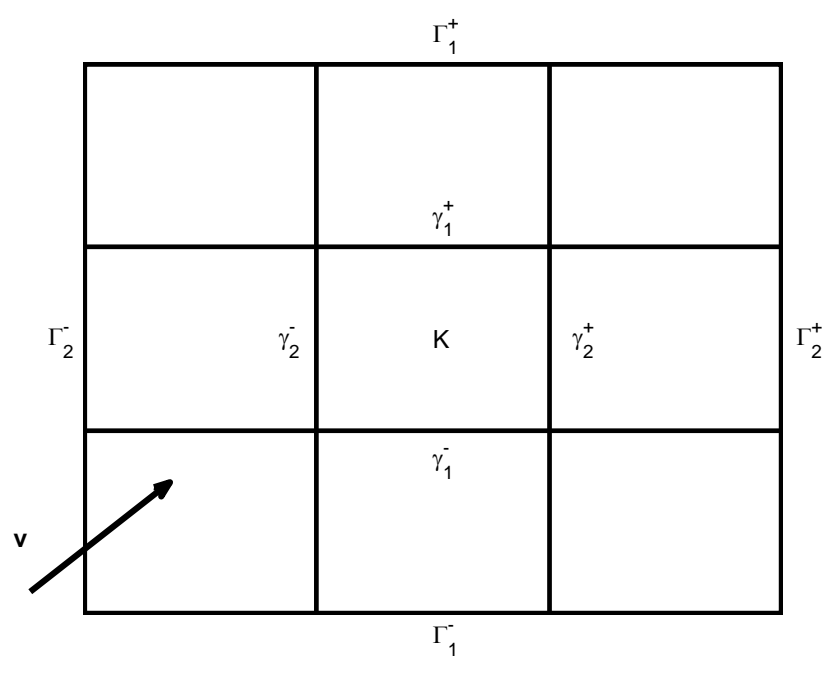

Fig. 2.1. An example of a Cartesian mesh $\mathbf{T}$ and a vector $\mathbf{v}$ to define mesh orientation with $\partial \Omega^{ \pm}=\Gamma_{1}^{ \pm} \cup \Gamma_{2}^{ \pm}$.

$$
\begin{gathered}
\hat{\mathbf{q}}_{N}=\left\{\begin{array}{ll}
\mathbf{q}_{N}^{+}-C_{11}\left(u_{N}^{+}-g_{D}\right) & \text { if } e \in \mathscr{E}_{D} \\
\mathbf{g}_{N} & \text { if } e \in \mathscr{E}_{N}
\end{array},\right. \\
\hat{u}_{N}=\left\{\begin{array}{ll}
u_{N}^{+} & \text {if } e \in \mathscr{E}_{N} \\
\mathscr{P}^{+} g_{D} & \text { if } e \in \mathscr{E}_{D}
\end{array},\right.
\end{gathered}
$$

Let $I=\left[a^{-}, a^{+}\right]$be an arbitrary interval, and let $P_{k}(I)$ be the space of polynomials of degree at most $k$ on $I$, the Radau projection $\mathscr{P}^{ \pm} w \in P_{k}(I)$ of $w$ is determined by the following $k+1$ conditions

$$
\int_{I}\left[w(x)-\mathscr{P}^{ \pm} w(x)\right] p(x) d x=0 \quad \forall p \in P_{k-1}(I), \quad \mathscr{P}^{ \pm} w\left(a^{ \pm}\right)=w\left(a^{ \pm}\right) .
$$

If $\mathscr{P}$ denotes the $L^{2}$ projection onto $P_{k}(I)$, then on a rectangle $K=I_{1} \times I_{2}$ with $I_{1}=\left[a^{-}, a^{+}\right]$and $I_{2}=\left[b^{-}, b^{+}\right]$and $\mathbf{r}=\left(r_{1}, r_{2}\right)^{t}$, we define $\Pi^{ \pm} \mathbf{r}=$ $\left(\mathscr{P}_{1}^{ \pm} \otimes \mathscr{P}_{2} r_{1}, \mathscr{P}_{1} \otimes \mathscr{P}_{2}^{ \pm} r_{2}\right)^{t}$ and $\pi^{ \pm} u=\mathscr{P}_{1}^{ \pm} \otimes \mathscr{P}_{2}^{ \pm} u$. The subscripts in $\mathscr{P}$ and $\mathscr{P}^{ \pm}$indicate the one dimensional operators, for instance $\mathscr{P}_{1}, \mathscr{P}_{1}^{+}, \mathscr{P}_{1}^{-}$, respectively, denote the projections $\mathscr{P}, \mathscr{P}^{+}, \mathscr{P}^{-}$onto $P_{k}\left(I_{1}\right)$ in the $x_{1}$ variable. Similarly, $\mathscr{P}_{2}, \mathscr{P}_{2}^{+}, \mathscr{P}_{2}^{-}$, respectively, denote the projections $\mathscr{P}, \mathscr{P}^{+}, \mathscr{P}^{-}$ onto $P_{k}\left(I_{2}\right)$ in the $x_{2}$ variable. Next we describe the two dimensional operator $\mathscr{P}_{1}^{+} \otimes \mathscr{P}_{2}$ applied to a scalar function $u\left(x_{1}, x_{2}\right)$ defined on the rectangle $K=$ $I_{1} \times I_{2}$. Let $p_{i}^{(1)}\left(x_{1}\right)$ and $p_{i}^{(2)}\left(x_{2}\right)$, respectively, denote the $i^{t h}$-degree Legendre 
polynomials shifted to $I_{1}$ and $I_{2}$ and apply the $L^{2}$ projection operator $\mathscr{P}_{2}$ to $u$ in the $x_{2}$ variable on $I_{2}$ to find $\mathscr{P}_{2} u\left(x_{1}, x_{2}\right)=\sum_{i=0}^{k} c_{i}\left(x_{1}\right) p_{i}^{(2)}\left(x_{2}\right)$ such that

$$
\int_{I_{2}}\left[u\left(x_{1}, x_{2}\right)-\mathscr{P}_{2} u\left(x_{1}, x_{2}\right)\right] p_{i}^{(2)}\left(x_{2}\right) d x_{2}=0, \quad \forall i=0,1, \ldots, k .
$$

Then, we apply $\mathscr{P}_{1}^{+}$to $\mathscr{P}_{2} u$ in the $x_{1}$ variable on $I_{1}$ to find $\mathscr{P}_{1}^{+} \otimes \mathscr{P}_{2} u\left(x_{1}, x_{2}\right)=$ $\sum_{i=0}^{k} \sum_{j=0}^{k} c_{i j} p_{j}^{(1)}\left(x_{1}\right) p_{i}^{(2)}\left(x_{2}\right) \in Q_{k}$ such that

$$
\int_{I_{1}}\left[\mathscr{P}_{2} u\left(x_{1}, x_{2}\right)-\mathscr{P}_{1}^{+} \otimes \mathscr{P}_{2} u\left(x_{1}, x_{2}\right)\right] p_{j}^{(1)}\left(x_{1}\right) d x_{1}=0, \forall j=0,1, \ldots, k-1,
$$

and

$$
\mathscr{P}_{1}^{+} \otimes \mathscr{P}_{2} u\left(a^{+}, x_{2}\right)=\mathscr{P}_{2} u\left(a^{+}, x_{2}\right) .
$$

Since $\mathbf{v}$ has strictly positive components we use the projections

$$
\left.\Pi \mathbf{q}\right|_{K}:=\left.\Pi^{-} \mathbf{q}\right|_{K},\left.\quad \pi u\right|_{K}:=\left.\pi^{+} u\right|_{K}, \quad \forall K \in \mathbf{T}
$$

having the properties

$$
\begin{aligned}
\int_{K}\left[\mathbf{r}-\boldsymbol{\Pi}^{-} \mathbf{r}\right] \cdot \nabla v d x & =0, \quad \forall v \in Q^{k}(K), \\
\int_{\gamma_{i}^{-}}\left[\mathbf{r}-\boldsymbol{\Pi}^{-} \mathbf{r}\right] \cdot \mathbf{n} v d s & =0, \quad \forall v \in Q^{k}\left(\gamma_{i}^{-}\right), i=1,2, \\
\left.\left(u-\pi^{+} u\right)\right|_{\gamma_{i}^{+}} & =\left.u\right|_{\gamma_{i}^{+}}-\left.\mathscr{P}_{i}^{+} u\right|_{\gamma_{i}^{+}}, i=1,2,
\end{aligned}
$$

where $\gamma_{i}^{+}, i=1,2$ are the outflow edges and $\gamma_{i}^{-}, i=1,2$ are inflow edges of element $K$ as illustrated in Figure 2.1.

In our analysis we need the a priori error estimates stated in the following Lemma.

Lemma 2.1 Let $v \in H^{s+2}(K)$ and $\mathbf{r} \in H^{s+1}(K)^{2}, s \geq 0$. Then for $m=0,1$, we have

$$
\begin{aligned}
\left|v-\pi^{ \pm} v\right|_{m, K} & \leq C h^{\min \{s+1, k\}+1-m}\|v\|_{s+2, K}, \\
\left\|v-\pi^{ \pm} v\right\|_{0, e} & \leq C h^{\min \{s+1, k\}+\frac{1}{2}}\|v\|_{s+2, K}, \quad \forall e \in \partial K \\
\left|\mathbf{r}-\boldsymbol{\Pi}^{ \pm} \mathbf{r}\right|_{m, K} & \leq C h^{\min \{s, k\}+1-m}\|\mathbf{r}\|_{s+1, K}, \\
\left\|\mathbf{r}-\boldsymbol{\Pi}^{ \pm} \mathbf{r}\right\|_{0, e} & \leq C h^{\min \{s, k\}+\frac{1}{2}}\|\mathbf{r}\|_{s+1, K}, \quad \forall e \in \partial K
\end{aligned}
$$

Furthermore, for any edge $e_{i}$ parallel to the $x_{i}$-axis, $i=1,2$, we have

$$
\left\|w-\mathscr{P}_{i}^{ \pm} w\right\|_{0, e_{i}} \leq C h^{\min \left\{s+\frac{1}{2}, k\right\}+1}\|w\|_{s+\frac{3}{2}, e_{i}}, \quad \forall w \in H^{s+\frac{3}{2}}\left(e_{i}\right) .
$$


If $v \in W^{s+1, \infty}(K)$, then

$$
\left\|v-\pi^{ \pm} v\right\|_{L^{\infty}(e)} \leq C h^{\min \{s, k\}+1}\|v\|_{W^{s+1, \infty}(K)}, \quad \forall e \in \partial K .
$$

Let $\mathscr{R}^{+} v$ be the $k$-degree polynomial interpolating $v$ at the roots of the $(k+1)$ degree right Radau polynomial shifted to the edge $e$ and $v \in H^{k+2}(e)$ then

$$
\left\|\mathscr{P}^{+} v-\mathscr{R}^{+} v\right\|_{0, e} \leq C h^{k+2}\|v\|_{k+2, e} .
$$

$A(\mathbf{q}, u ; \mathbf{r}, v):=a(\mathbf{q}, \mathbf{r})+b(u, \mathbf{r})-b(v, \mathbf{q})+c(u, v), \quad \mathscr{F}(\mathbf{r}, v):=F(\mathbf{r})+G(v)$ and

$$
\begin{gathered}
a(\mathbf{q}, \mathbf{r})=\int_{\Omega} \mathbf{q} \cdot \mathbf{r} d x \\
b(u, \mathbf{r})=\sum_{K \in \mathbf{T}} \int_{K} u \nabla \cdot \mathbf{r} d x-\sum_{e \in \mathscr{E}_{I}} \int_{e}\left(\{u\}+\mathbf{C}_{12} \llbracket u \rrbracket\right) \llbracket \mathbf{r} \rrbracket d s-\sum_{e \in \mathscr{E}_{N}} \int_{e} u \mathbf{r} \cdot \mathbf{n} d s \\
c(u, v)=\sum_{e \in \mathscr{E}_{D}^{+}} \int_{e} C_{11} u v d s \\
F(\mathbf{r})=\sum_{e \in \mathscr{E}_{D}} \int_{e} \mathscr{P}^{+} g_{D} \mathbf{r} \cdot \mathbf{n} d s
\end{gathered}
$$




$$
G(v)=\int_{K} f v d x+\sum_{e \in \mathscr{E}_{D}^{+}} \int_{e} C_{11} v g_{D} d s+\sum_{e \in \mathscr{E}_{N}} \int_{e} v \mathbf{g}_{N} \cdot \mathbf{n} d s
$$

We note the use of $\mathscr{P}^{+} g_{D}$ in the right hand side $F$ is essential for obtaining superconvergence of the solution gradient q everywhere. However, The use of either of $\mathscr{P}^{+} g_{D}$ or $g_{D}$ in the right hand side $G$ yields superconvergence for $\mathbf{q}$. Furthermore, we note that the true solution satisfies

$$
\begin{array}{r}
A(\mathbf{q}, u ; \mathbf{r}, v)=a(\mathbf{q}, \mathbf{r})+b(u, \mathbf{r})-b(v, \mathbf{q})+c(u, v)=\int_{K} f v d x+\int_{\partial \Omega_{D}^{+}} C_{11} g_{D} v d s+ \\
\int_{\partial \Omega_{D}} g_{D} \mathbf{r} \cdot \mathbf{n} d s+\int_{\partial \Omega_{N}} v \mathbf{g}_{N} \cdot \mathbf{n} d s, \quad \forall(\mathbf{q}, u) \in\left(H^{1}(\Omega)\right)^{2} \times H^{2}(\Omega) .
\end{array}
$$

Subtracting (2.25a) from (2.26), and letting $\xi_{u}=u-\pi u, \xi_{\mathbf{q}}=\mathbf{q}-\Pi \mathbf{q}$, $e_{u}=u-u_{N}, \mathbf{e}_{\mathbf{q}}=\mathbf{q}-\mathbf{q}_{N}, \pi e_{u}=\pi u-u_{N}$ and $\boldsymbol{\Pi}_{\mathbf{q}}=\Pi \mathbf{q}-\mathbf{q}_{N}$, the following LDG orthogonality condition holds for all $(\mathbf{r}, v) \in M_{N} \times V_{N}$

$$
A\left(\mathbf{e}_{\mathbf{q}}, e_{u} ; \mathbf{r}, v\right)-\sum_{e \in \mathscr{E}_{D}} \int_{e}\left(g_{D}-\mathscr{P}^{+} g_{D}\right) \mathbf{r} \cdot \mathbf{n} d s=0
$$

In the remainder of this manuscript we use the semi norm

$$
|(\mathbf{q}, u)|_{A}^{2}=A(\mathbf{q}, u ; \mathbf{q}, u)=\|\mathbf{q}\|_{0}^{2}+C_{11}\|u\|_{0, \partial \Omega_{D}^{+}}^{2},
$$

and assume

$$
\partial \Omega_{N} \cap \partial \Omega^{-}=\emptyset
$$

The next lemma contains preliminary results needed in our analysis.

Lemma 2.2 Let $u \in H^{k+2}(\Omega), s \geq 0$, and set $\mathbf{q}=\nabla u$. Assume further that $\mathbf{q} \in W^{k+1, \infty}$ and that $C_{11}$ is a positive constant, and let $\boldsymbol{\Pi}$ and $\pi$ be the operators defined in (2.21). Then, for all $(\mathbf{r}, v) \in M_{N} \times V_{N}$, we have

$$
\left|A\left(-\xi_{\mathbf{q}},-\xi_{u} ; \mathbf{r}, v\right)+\sum_{e \in \mathscr{E}_{D}} \int_{e}\left(g_{D}-\mathscr{P}^{+} g_{D}\right) \mathbf{r} \cdot \mathbf{n} d s\right| \leq C h^{k+1}|(\mathbf{r}, v)|_{A}
$$

Furthermore, if $\varphi \in H^{2}$ such that $\left.\varphi\right|_{\partial \Omega_{D}}=0$, and $\Phi=\nabla \varphi$ we have

$$
|A(\mathbf{r}, v ; \Phi-\Pi \Phi, \varphi-\pi \varphi)| \leq C|(\mathbf{r}, v)|_{A}|| \varphi \|_{2},
$$

where $C>0$ is a generic constant independent of $h, v, \mathbf{r}$. 
Proof. First, we write

$$
\begin{aligned}
& A\left(-\xi_{\mathbf{q}},-\xi_{u} ; \mathbf{r}, v\right)+\sum_{e \in \mathscr{E}_{D}} \int_{e}\left(g_{D}-\mathscr{P}^{+} g_{D}\right) \mathbf{r} \cdot \mathbf{n} d s \\
& =a\left(-\xi_{\mathbf{q}}, \mathbf{r}\right)+b\left(-\xi_{u}, \mathbf{r}\right)-b\left(v,-\xi_{\mathbf{q}}\right)+c\left(-\xi_{u}, v\right) \\
& +\sum_{e \in \mathscr{E}_{D}} \int_{e}\left(g_{D}-\mathscr{P}^{+} g_{D}\right) \mathbf{r} \cdot \mathbf{n} d s \\
& =T_{1}+T_{2}+T_{3}+T_{4},
\end{aligned}
$$

where

$$
\begin{aligned}
& T_{1}=a\left(-\xi_{\mathbf{q}}, \mathbf{r}\right) \\
& T_{2}=-b\left(v,-\xi_{\mathbf{q}}\right), \\
& T_{3}=b\left(-\xi_{u}, \mathbf{r}\right)+\sum_{e \in \mathscr{E}_{D}} \int_{e}\left(g_{D}-\mathscr{P}^{+} g_{D}\right) \mathbf{r} \cdot \mathbf{n} d s \\
& T_{4}=-\sum_{e \in \mathscr{E}_{D}^{+}} \int_{e} C_{11} \xi_{u} v d s .
\end{aligned}
$$

Applying Cauchy Schwarz inequality to $T_{1}=a\left(-\xi_{\mathbf{q}}, r\right)$ leads to

$$
\begin{aligned}
\left|T_{1}\right| & \leq\left|\sum_{K \in \mathbf{T}} \int_{K} \mathbf{r} \cdot \xi_{\mathbf{q}} d x\right| \leq\left(\sum_{K \in \mathbf{T}}\|\mathbf{r}\|_{0, K}^{2}\right)^{1 / 2}\left(\sum_{K \in \mathbf{T}}\left\|\xi_{\mathbf{q}}\right\|_{0, K}^{2}\right)^{1 / 2} \\
& \leq C|(\mathbf{r}, v)|_{A}\left(\sum_{K \in \mathbf{T}} h^{2 k+2}\|\mathbf{q}\|_{k+1, K}^{2}\right)^{1 / 2}
\end{aligned}
$$

Next, we integrate $b\left(v,-\xi_{\mathbf{q}}\right)$ in $(2.25 \mathrm{~d})$ by parts to write $T_{2}$

$$
T_{2}=-b\left(v,-\xi_{\mathbf{q}}\right)=-\sum_{K \in \mathbf{T}} \int_{K} \nabla v \cdot \xi_{\mathbf{q}} d x+\sum_{e \in \mathscr{E}_{D}} \int_{e} v \xi_{\mathbf{q}} \cdot \mathbf{n} d s+\sum_{e \in \mathscr{E}_{I}} \int_{e} \llbracket v \rrbracket \hat{\xi}_{\mathbf{q}} d s
$$

where $\hat{\xi}_{\mathbf{q}}$ on an interior edge is the limit of $\xi_{\mathbf{q}}$ from the right on a vertical edge $\therefore$ and the limit from above on a horizontal edge.

Using the properties $(2.22)$ of the projection $\boldsymbol{\Pi}^{-}$, we obtain

$$
\begin{gathered}
\int_{K} \nabla v \cdot \xi_{\mathbf{q}} d x=0, \quad \forall K \in T, \quad \int_{e} v \xi_{\mathbf{q}} \cdot \mathbf{n} d s=0, \quad \forall e \subset \partial \Omega^{-}, \\
\int_{e} \llbracket v \rrbracket \cdot \hat{\xi}_{\mathbf{q}} d s=0, \quad \forall e \in \mathscr{E}_{I} .
\end{gathered}
$$

Hence,

$$
T_{2}=\sum_{e \in \mathscr{E}_{D}^{+}} \int_{e} v \xi_{\mathbf{q}} \cdot \mathbf{n} d s
$$


Multiplying and dividing each term of the sum by $C_{11}^{1 / 2}$ and applying Cauchy Schwarz inequality, we obtain

$$
\begin{gathered}
\left|T_{2}\right|=\left|\sum_{e \in \mathscr{E}_{D}^{+}} \int_{e} v \xi_{\mathbf{q}} \cdot \mathbf{n} d s\right| \leq\left(\sum_{e \in \mathscr{E}_{D}^{+}} C_{11} \mid\|v\|_{0, e}^{2}\right)^{1 / 2}\left(\sum_{e \in \mathscr{E}_{D}^{+}} C_{11}^{-1}\left\|\xi_{\mathbf{q}}\right\|_{0, e}^{2}\right)^{1 / 2} \\
(\text { by Lemma } 2.1) \leq C|(\mathbf{r}, v)|_{A}\left(\sum_{e \in \mathscr{E}_{D}^{+}} h^{2 k+1}\|\mathbf{q}\|_{k+1, K}^{2}\right)^{1 / 2} \\
\leq C|(\mathbf{r}, v)|_{A}\left(\sum_{e \in \partial \Omega_{D}^{+}} h^{2 k+1} h^{2}\|\mathbf{q}\|_{W^{k+1, \infty}}^{2}\right)^{1 / 2} \\
\leq C|(\mathbf{r}, v)|_{A}\left(\sum_{e \in \mathscr{E}_{D}^{+}} h^{2 k+3}\right)^{1 / 2}\|\mathbf{q}\|_{W^{k+1, \infty}} \\
=C|(\mathbf{r}, v)|_{A} h^{k+1}\|\mathbf{q}\|_{W^{k+1, \infty}} .
\end{gathered}
$$

For $T_{3}$ we write

$$
\begin{gathered}
T_{3}=b\left(-\xi_{u}, \mathbf{r}\right)+\sum_{e \in \mathscr{E}_{D}} \int_{e}\left(g_{D}-\mathscr{P}^{+} g_{D}\right) \mathbf{r} \cdot \mathbf{n} d s= \\
-\left(\sum_{K \in \mathbf{T}} \xi_{u} \nabla \cdot \mathbf{r} d x-\sum_{K \in \mathbf{T}}\left(\sum_{\substack{e \in \mathscr{E}_{I} \\
e \subset \partial K}} \int_{e} \hat{\xi}_{u} \mathbf{r} \cdot \mathbf{n} d s\right)-\sum_{e \in \mathscr{E}_{N}} \int_{e} \xi_{u} \mathbf{r} \cdot \mathbf{n} d s\right)+ \\
\sum_{e \in \mathscr{E}_{D}} \int_{e}\left(g_{D}-\mathscr{P} g_{D}\right) \mathbf{r} \cdot \mathbf{n} d s
\end{gathered}
$$

89 where $\hat{\xi}_{u}$ on an interior edge is the limit of $\xi_{u}$ from the left if the edge is vertical and from the bottom if the edge is horizontal.

Using the assumption (2.29) we write

$$
\begin{aligned}
T_{3} & =-\left(\sum_{K \in \mathbf{T}} \xi_{u} \nabla \cdot \mathbf{r} d x-\sum_{K \in \mathbf{T}}\left(\sum_{\substack{e \in \mathscr{E}_{I} \\
e \subset \partial K}} \int_{e}\left(u-\mathscr{P}^{+} u\right) \mathbf{r} \cdot \mathbf{n} d s\right)\right) \\
& +\sum_{e \subset \partial \Omega} \int_{e}\left(u-\mathscr{P}^{+} u\right) \mathbf{r} \cdot \mathbf{n} d s \\
& =-\sum_{K \in \mathbf{T}} Z_{K}(\mathbf{r}, u),
\end{aligned}
$$


where

$$
Z_{K}(\mathbf{r}, u)=\int_{K} \xi_{u} \nabla \cdot \mathbf{r} d x-\int_{\gamma_{1}^{-} \cup \gamma_{1}^{+}}\left(u-\mathscr{P}_{1}^{+} u\right) \mathbf{r} \cdot \mathbf{n} d s-\int_{\gamma_{2}^{-} \cup \gamma_{2}^{+}}\left(u-\mathscr{P}_{2}^{+} u\right) \mathbf{r} \cdot \mathbf{n} d s,
$$

Applying Cauchy Schwarz inequality and Lemma 3.6 from [9] which proves that if $u \in H^{k+2}(\Omega)$ and $\mathbf{r} \in\left(H^{1}(\Omega)\right)^{2}$, then $Z_{K}(\mathbf{r}, u)$ can be bounded as

$$
\left|Z_{K}(\mathbf{r}, u)\right| \leq C h^{k+1}\|u\|_{k+2, K}\|\mathbf{r}\|_{0, K},
$$

we write

$$
\sum_{K \in \mathbf{T}} Z_{K}(\mathbf{r}, u) \leq C \sum_{K \in \mathbf{T}} h^{k+1}\|u\|_{k+2, K}|| \mathbf{r}\left\|_{0, K} \leq C|(\mathbf{r}, v)|_{A} h^{k+1}\right\| u \|_{k+2} .
$$

Thus,

$$
\left|T_{3}\right| \leq\left. C|(\mathbf{r}, v)|_{A} h^{k+1}|| u\right|_{k+2} .
$$

Applying Cauchy Schwartz inequality we write

$$
\begin{aligned}
\left|T_{4}\right| & \leq\left|\sum_{e \in \mathscr{E}_{D}^{+}} \int_{e} C_{11} \xi_{u} v d s\right| \\
& \leq\left(\sum_{e \in \mathscr{E}_{D}^{+}} C_{11}\|v\|_{0, e}^{2}\right)^{1 / 2}\left(\sum_{e \in \mathscr{E}_{D}^{+}} C_{11}\left\|\xi_{u}\right\|_{0, e}^{2}\right)^{1 / 2},
\end{aligned}
$$

leading to

$$
\left|T_{4}\right| \leq|(\mathbf{r}, v)|_{A}\left(\sum_{e \in \mathscr{E}_{D}^{+}} C_{11}|| \xi_{u} \|_{0, e}^{2}\right)^{1 / 2}
$$

which, by Lemma 2.1, becomes

$$
\begin{aligned}
\left|T_{4}\right| & \leq|(\mathbf{r}, v)|_{A} C\left(\sum_{e \in \mathscr{E}_{D}^{+}} h^{2 k+1}|| u \|_{k+2, K}^{2}\right)^{1 / 2} \\
& \leq|(\mathbf{r}, v)|_{A} C h^{k+1}\|u\|_{W^{k+2, \infty}} .
\end{aligned}
$$

The proof of (2.30) is completed by combining the estimates for $T_{1}, T_{2}, T_{3}$ and $T_{4}$.

Similarly, we establish (2.31) by splitting

$$
\begin{aligned}
A(\mathbf{r}, v ; \boldsymbol{\Phi}-\Pi \Phi, \varphi-\pi \varphi) & =a(\mathbf{r}, \boldsymbol{\Phi}-\Pi \mathbf{\Phi})+b(v, \boldsymbol{\Phi}-\Pi \mathbf{\Phi})-b(\varphi-\pi \varphi, \mathbf{r}) \\
& +c(v, \varphi-\pi \varphi) \\
& =\tilde{T}_{1}+\tilde{T}_{2}+\tilde{T}_{3}+\tilde{T}_{4},
\end{aligned}
$$

where

$$
\tilde{T}_{1}=a\left(\mathbf{r}, \xi_{\boldsymbol{\Phi}}\right), \tilde{T}_{2}=-b\left(v, \xi_{\boldsymbol{\Phi}}\right), \tilde{T}_{3}=b\left(\xi_{\varphi}, \mathbf{r}\right), \tilde{T}_{4}=\sum_{e \in \mathscr{E}_{D}^{+}} \int_{e} C_{11} \xi_{\varphi} v d s .
$$


${ }_{97}$ We establish the proof by bounding each of terms $\tilde{T}_{i}, i=1,2,3,4$.

98 Following (2.34) for $T_{1}$ we apply Cauchy Schwarz inequality to $\tilde{T}_{1}$ and Lemma

$99 \quad 2.1$ to obtain

$$
\left|\tilde{T}_{1}\right| \leq|(\mathbf{r}, v)|_{A}\left(\sum_{K \in \mathbf{T}}\left\|\xi_{\boldsymbol{\Phi}}\right\|_{0, K}^{2}\right)^{1 / 2} \leq|(\mathbf{r}, v)|_{A}\left\|\xi_{\boldsymbol{\Phi}}\right\|_{0} \leq C|(\mathbf{r}, v)|_{A}\|\varphi\|_{2}
$$

We follow (2.35) and (2.36) for $T_{2}$ and use projection properties (2.22) and Lemma 2.1 to write

$$
\begin{aligned}
\left|\tilde{T}_{2}\right| & =\left|\sum_{e \in \mathscr{E}_{D}^{+}} \int_{e} v \xi_{\boldsymbol{\Phi}} \cdot \mathbf{n} d s\right| \\
& \leq C|(\mathbf{r}, v)|_{A}\left(\sum_{e \in \mathscr{E}_{D}^{+}} C_{11}^{-1}|| \xi_{\boldsymbol{\Phi}} \|_{0, e}^{2}\right)^{1 / 2} \\
& \leq C|(\mathbf{r}, v)|_{A}\|\varphi\|_{2} .
\end{aligned}
$$

Next we will find a bound for $\tilde{T}_{3}$ as

$$
\left|\tilde{T}_{3}\right|=\left|-\left(\sum_{K \in \mathbf{T}} \xi_{\varphi} \nabla \cdot \mathbf{r} d x-\sum_{K \in \mathbf{T}}\left(\sum_{e \in \mathscr{E}_{I}, e \subset \partial K} \int_{e} \hat{\xi}_{\varphi} \mathbf{r} \cdot \mathbf{n} d s\right)-\sum_{e \in \mathscr{E}_{N}} \int_{e} \xi_{\varphi} \mathbf{r} \cdot \mathbf{n} d s\right)\right|,
$$




$$
\left|\left(\boldsymbol{\Pi}_{\mathbf{q}}, \Pi e_{u}\right)\right|_{A}^{2}=A\left(-\xi_{\mathbf{q}},-\xi_{u} ; \Pi \mathbf{e}_{\mathbf{q}}, \pi e_{u}\right)+\sum_{e \in \mathscr{E}_{D}} \int_{e}\left(g_{D}-\mathscr{P}^{+} g_{D}\right) \boldsymbol{\Pi} \mathbf{e}_{\mathbf{q}} \cdot \mathbf{n} d s
$$

Applying Lemma 2.2 yields

$$
\left.\mid A\left(-\xi_{\mathbf{q}},-\xi_{u} ; \Pi \mathbf{e}_{\mathbf{q}}, \pi e_{u}\right)\right)+\left.\sum_{e \in \mathscr{E}_{D}} \int_{e}\left(g_{D}-\mathscr{P}^{+} g_{D}\right) \boldsymbol{\Pi} \mathbf{e}_{\mathbf{q}} \cdot \mathbf{n} d s\left|\leq C h^{k+1}\right|\left(\boldsymbol{\Pi} \mathbf{e}_{\mathbf{q}}, \Pi e_{u}\right)\right|_{A},
$$

which establishes

$$
\left|\left(\boldsymbol{\Pi e}_{\mathbf{q}}, \pi e_{u}\right)\right|_{A} \leq C h^{k+1} .
$$

${ }_{112}$ We complete the proof of (2.44) by combining (2.46), (2.47) and (2.50). 
114

$$
\begin{aligned}
-\Delta \varphi & =\lambda, & & \text { in } \Omega, \\
\varphi & =0, & & \text { on } \partial \Omega_{D}, \\
\frac{\partial \varphi}{\partial n} & =0, & & \text { on } \partial \Omega_{N} .
\end{aligned}
$$

One can verify that for $\mathbf{s} \in\left(L^{2}\right)^{2}$ and $w \in L^{2}$ the following holds

$$
A(-\mathbf{\Phi}, \varphi ;-\mathbf{s}, w)=(\lambda, w)=\int_{\Omega} \lambda w d x .
$$

Letting $\mathbf{s}=\mathbf{e}_{\mathbf{q}}$, and $w=\lambda=e_{u}$ we obtain

$$
A\left(-\boldsymbol{\Phi}, \varphi ;-\mathbf{e}_{\mathbf{q}}, e_{u}\right)=\left\|e_{u}\right\|_{0}^{2},
$$

which leads to

$$
\left\|e_{u}\right\|_{0}^{2}=A\left(\mathbf{e}_{\mathbf{q}}, e_{u} ; \boldsymbol{\Phi}, \varphi\right)
$$

117 Applying the orthogonality condition (2.27) yields

$$
\left\|e_{u}\right\|_{0}^{2}=A\left(\mathbf{e}_{\mathbf{q}}, e_{u} ; \boldsymbol{\Phi}-\boldsymbol{\Pi} \boldsymbol{\Phi}, \varphi-\pi \varphi\right)+\sum_{e \in \mathscr{E}_{D}} \int_{e}\left(g_{D}-\mathscr{P}^{+} g_{D}\right) \boldsymbol{\Pi} \boldsymbol{\Phi} \cdot \mathbf{n} d s
$$

$$
\begin{aligned}
\left\|e_{u}\right\|_{0}^{2}=A\left(\Pi \mathbf{e}_{\mathbf{q}}, \pi e_{u} ; \boldsymbol{\Phi}-\Pi \boldsymbol{\Phi}, \varphi-\pi \varphi\right)+A\left(\xi_{q}, \xi_{u} ; \boldsymbol{\Phi}-\Pi \mathbf{\Phi}, \varphi-\pi \varphi\right) \\
+\sum_{e \in \mathscr{E}_{D}} \int_{e}\left(g_{D}-\mathscr{P}^{+} g_{D}\right) \Pi \boldsymbol{\Pi} \cdot \mathbf{n} d s
\end{aligned}
$$

which can be written as

$$
\begin{aligned}
\left\|e_{u}\right\|_{0}^{2} & =A\left(\mathbf{\Pi e}_{\mathbf{q}}, \pi e_{u} ; \mathbf{\Phi}-\boldsymbol{\Pi} \mathbf{\Phi}, \varphi-\pi \varphi\right) \\
& -\left[A\left(-\xi_{q},-\xi_{u} ; \boldsymbol{\Phi}-\boldsymbol{\Pi} \boldsymbol{\Phi}, \varphi-\pi \varphi\right)+\sum_{e \in \mathscr{E}_{D}} \int_{e}\left(g_{D}-\mathscr{P}^{+} g_{D}\right)(\mathbf{\Phi}-\boldsymbol{\Pi} \boldsymbol{\Phi}) \cdot \mathbf{n} d s\right] \\
& +\sum_{e \in \mathscr{E}_{D}} \int_{e}\left(g_{D}-\mathscr{P}^{+} g_{D}\right) \boldsymbol{\Phi} \cdot \mathbf{n} d s \\
& =H_{1}+H_{2}+H_{3},
\end{aligned}
$$


where

$$
\begin{aligned}
& H_{1}=A\left(\Pi \mathbf{e}_{\mathbf{q}}, \pi e_{u} ; \boldsymbol{\Phi}-\Pi \mathbf{\Phi}, \varphi-\pi \varphi\right), \\
& H_{2}=-\left[A\left(-\xi_{q},-\xi_{u} ; \boldsymbol{\Phi}-\Pi \mathbf{\Phi}, \varphi-\pi \varphi\right)+\sum_{e \in \mathscr{E}_{D}} \int_{e}\left(g_{D}-\mathscr{P}^{+} g_{D}\right)(\mathbf{\Phi}-\Pi \mathbf{\Phi}) \cdot \mathbf{n} d s\right], \\
& H_{3}=\sum_{e \in \mathscr{E}_{D}} \int_{e}\left(g_{D}-\mathscr{P}^{+} g_{D}\right) \boldsymbol{\Phi} \cdot \mathbf{n} d s .
\end{aligned}
$$

Applying Lemma 2.2 and the estimate (2.50) we prove that

$$
\left|H_{1}\right| \leq C\left|\left(\boldsymbol{\Pi e}_{\mathbf{q}}, \pi e_{u}\right)\right|_{A}\|\varphi\|_{2} \leq C h^{k+1}\left\|e_{u}\right\|_{0}
$$

By Lemma 2.2

$$
\left|H_{2}\right| \leq C h^{k+1}|(\mathbf{\Phi}-\Pi \mathbf{\Phi}, \varphi-\pi \varphi)|_{A} \leq C h^{k+1}\|\varphi\|_{2} \leq C h^{k+1}|| e_{u} \|_{0} .
$$

Applying Cauchy Schwarz inequality, the trace theorem [5] and Lemma 2.1 we write

$$
\begin{gathered}
\left|H_{3}\right| \leq C\left\|g_{D}-\mathscr{P}^{+} g_{D}\right\|_{0, \partial \Omega_{D}}\|\Phi\|_{0, \partial \Omega_{D}} \leq C\left\|g_{D}-\mathscr{P}^{+} g_{D}\right\|_{0, \partial \Omega_{D}}\|\varphi\|_{2} \\
\leq C h^{k+1}\left\|e_{u}\right\|_{0} .
\end{gathered}
$$

Instead of the projection $\mathscr{P}^{+} g_{D}$, one could also use the interpolation $\mathscr{R}^{+} g_{D}$ of the boundary condition $g_{D}$ in (2.16) and still have $\mathcal{O}\left(h^{k+1}\right)$ superconvergence rates for both $u$ and $\mathbf{q}$. The proof will follow the same line of reasoning to prove Lemma 2.2 and Theorem 2.1. The term $T_{3}$ in the proof of Lemma 2.2 now contains

$$
\sum_{e \in \mathscr{E}_{D}} \int_{e}\left(g_{D}-\mathscr{R}^{+} g_{D}\right) \mathbf{r} \cdot \mathbf{n} d s .
$$

Adding and subtracting $\sum_{e \in \mathscr{E}_{D}} \int_{e} \mathscr{P}^{+} g_{D} \mathbf{r} \cdot \mathbf{n} d s$, we write $T_{3}$ as

$$
T_{3}=-\sum_{K \in \mathbf{T}} Z_{k}(\mathbf{r}, v)+\sum_{e \in \mathscr{E}_{D}} \int_{e}\left(\mathscr{P}^{+} g_{D}-\mathscr{R}^{+} g_{D}\right) \mathbf{r} \cdot \mathbf{n} d s
$$

Applying the bounds for $Z_{K}$ used above, the superconvergence result (2.24)

Letting $\mathscr{E}_{S} \subset\left(\mathscr{E} \backslash \mathscr{E}_{D}^{+}\right)$contain $\mathcal{O}(n)$ edges of a Cartesian mesh having $N=$ $n \times n$ elements, our proof can be extended to more general LDG methods with $C_{11}=\mathcal{O}(1)$ on all edges in $\mathscr{E}_{D}^{+} \cup \mathscr{E}_{S}$ and $C_{11}=0$ elsewhere. Hence, The terms 
$T_{1}, T_{2}$ and $T_{3}$ in (2.33) are unchanged while we obtain a new term $T_{4}$ as

$$
T_{4}=-\sum_{e \in \mathcal{E}_{S}} \int_{e} C_{11} \llbracket \xi_{u} \rrbracket \llbracket v \rrbracket d s-\sum_{e \in \mathscr{E}_{D}^{+}} \int_{e} C_{11} \xi_{u} v d s .
$$

Using the same line of reasoning we can establish a bound similar to (2.40). Moreover, one can easily prove the superconvergence of $\mathbf{q}_{N}$ and $u_{N}$ if $C_{11}=$ $\mathcal{O}(1)$ on all edges in $\mathscr{E}_{D}^{+} \cup \mathscr{E}_{S}$ and $C_{11}=h$ elsewhere. Numerical computations suggest that the superconvergence result holds for the case $C_{11}=1$ on all edges, however, we are not able to extend our error analysis to this case.

Finally, we note that this error analysis can be easily extended to three dimensional problems on hexahedral meshes.

\section{$3 \quad$ Numerical results}

In order to verify the sharpness of our $L^{2}$ a priori error estimates in Theorem 2.1 we conduct several computational experiments on the standard linear diffusion problem

$$
-\Delta u=f, \quad \in(-1,1)^{2},
$$

with Dirichlet boundary conditions and a source term $f(x, y)$ such that the true solution is

$$
u(x, y)=\cos (\pi x)+\cos (\pi y) .
$$

In all numerical experiments we use uniform Cartesian meshes having $N=$ $n^{2}=16,36,64,100,144,196,256,324,400$ square elements with spaces $Q_{k}$, $k=1,2,3,4$ to compute numerical solutions by several versions of LDG methods with the auxiliary vector $\mathbf{v}=[1,1]^{T}$.

First, we use the md-LDG method with stabilization parameter $C_{11}=1$, the projection $\mathscr{P}^{+} g_{D}$ in (2.16) and $g_{D}$ in (2.15). In Table 3.1 we present the mdLDG $L^{2}$ errors for $u$ and $\mathbf{q}$ which are in full agreement with Theorem 2.1 and yield $\mathcal{O}\left(h^{k+1}\right)$ convergence rates. We repeat the previous computational experiment using the true Dirichlet boundary condition $g_{D}$ in $(2.16)$ and $\mathscr{P}^{+} g_{D}$ in (2.15) and present the md-LDG $L^{2}$ errors for $u$ and the first component of $\mathbf{q}$ in Table 3.2. In this case we obtain the suboptimal $\mathcal{O}\left(h^{k+1 / 2}\right)$ convergence rates for $\mathbf{q}$ which confirms our error analysis that states that $\mathscr{P}^{+} g_{D}$ is only needed in (2.16). Next, we use the LDG method with $C_{11}=1$ on all edges in $\mathscr{E}_{D}^{+}$and $C_{11}=h$ elsewhere with the projection $\mathscr{P}^{+} g_{D}$ used in (2.16) and (2.15). We present the $L^{2}$ errors in Table 3.3 which are in full agreement with our theory. We repeat this experiment using the LDG method with $C_{11}=1$ on all edges in $\mathscr{E}_{D}^{+} \cup \mathscr{E}_{S}$ where $\mathscr{E}_{S}$ is the set of all edges on the lines $x=-1+6 / n$ 
and $y=-1+6 / n$ and $C_{11}=0$ elsewhere and show $L^{2}$ errors in Table 3.4. Again, these results are in full agreement with our theory.

The final case is not covered by our theory and consists of the LDG method with $C_{11}=1$ on all edges with the projection $\mathscr{P}^{+} g_{D}$ applied in (2.16) and (2.15). The $L^{2}$ errors and their orders of convergence shown in Table 3.5 also suggest optimal $\mathcal{O}\left(h^{k+1}\right)$ convergence rates for both $u$ and $\mathbf{q}$.

\section{Conclusion}

We proved that using a suitable projection or interpolation of the Dirichlet boundary data the LDG solutions for both $u$ and its gradient $\mathbf{q}$ converge at $\mathcal{O}\left(h^{k+1}\right)$ on regular Cartesian meshes with $k$-degree tensor product polynomial spaces. This is an improvement over existing $\mathcal{O}\left(h^{k+1 / 2}\right)$ results for q. Extensive numerical computations suggest that similar $\mathcal{O}\left(h^{k+1}\right)$ convergence rates also hold the LDG method with $C_{11}=1$ on all edges for elliptic problems and the md-LDG for convection-diffusion problems [3], however, our analysis does not extend to these cases and will be subject to further investigation. Moreover, numerical experiments [2,3] for modified spaces $\mathcal{V}_{k}=P_{k+1} \cap Q_{k}$ also exhibit $\mathcal{O}\left(h^{k+1}\right)$ superconvergence rates for both $u$ and $\mathbf{q}$ which is yet to be proved.

\section{References}

[1] S. Adjerid, M. Baccouch, Asymptotically exact a posteriori error estimates for a one-dimensional linear hyperbolic problem, Appl. Numer. Math. 60 (2010) 903-914. URL http://dx.doi.org/10.1016/j.apnum.2010.04.014

[2] S. Adjerid, M. Baccouch, A superconvergent local discontinuous Galerkin method for elliptic problems, J. Sci. Comput. 52 (2012) 113-152. URL http://dx.doi.org/10.1007/s10915-011-9537-8

[3] S. Adjerid, M. Baccouch, A posteriori local discontinuous Galerkin error estimation for two-dimensional convection-diffusion problems, J. Sci. Comput.In press.

URL http://dx.doi.org/10.1007/s10915-014-9861-x

[4] F. Bassi, S. Rebay, A high-order accurate discontinuous finite element method for the numerical solution of the compressible Navier-Stokes equations, J. Comput. Phys. 131 (1997) 267-279. URL http://dx.doi.org/10.1006/jcph.1996.5572

[5] S. Brenner, R. Scott, The Mathematical Theory of Finite Element Methods, Springer, New York, 2008. 


\begin{tabular}{|c|c|c|c|c|c|}
\hline$k$ & $N$ & $\left\|u-u_{N}\right\|_{0, \Omega}$ & Order & $\left\|q_{1}-q_{1, N}\right\|_{0, \Omega}$ & Order \\
\hline \multirow{9}{*}{$k=1$} & 16 & $4.4127 \mathrm{e}-01$ & NA & $8.0770 \mathrm{e}-01$ & NA \\
\hline & 36 & $1.4973 \mathrm{e}-01$ & 2.6656 & $3.2685 \mathrm{e}-01$ & 2.2313 \\
\hline & 64 & $7.5654 \mathrm{e}-02$ & 2.3729 & $1.7566 \mathrm{e}-01$ & 2.1583 \\
\hline & 100 & $4.6725 \mathrm{e}-02$ & 2.1596 & $1.0996 \mathrm{e}-01$ & 2.0992 \\
\hline & 144 & $3.2141 \mathrm{e}-02$ & 2.0521 & $7.5482 \mathrm{e}-02$ & 2.0636 \\
\hline & 196 & $2.3605 \mathrm{e}-02$ & 2.0026 & $5.5094 \mathrm{e}-02$ & 2.0425 \\
\hline & 256 & $1.8119 \mathrm{e}-02$ & 1.9805 & $4.2016 \mathrm{e}-02$ & 2.0295 \\
\hline & 324 & $1.4365 \mathrm{e}-02$ & 1.9712 & $3.3115 \mathrm{e}-02$ & 2.0211 \\
\hline & 400 & $1.1675 \mathrm{e}-02$ & 1.9680 & $2.6780 \mathrm{e}-02$ & 2.0155 \\
\hline \multirow{9}{*}{$k=2$} & 16 & $8.2880 \mathrm{e}-02$ & NA & $1.0980 \mathrm{e}-01$ & NA \\
\hline & 36 & $2.5288 \mathrm{e}-02$ & 2.9277 & $3.5270 \mathrm{e}-02$ & 2.8009 \\
\hline & 64 & $1.0205 \mathrm{e}-02$ & 3.1542 & $1.4991 \mathrm{e}-02$ & 2.9741 \\
\hline & 100 & $4.9612 \mathrm{e}-03$ & 3.2324 & $7.6106 \mathrm{e}-03$ & 3.0380 \\
\hline & 144 & $2.7357 \mathrm{e}-03$ & 3.2649 & $4.3513 \mathrm{e}-03$ & 3.0664 \\
\hline & 196 & $1.6503 \mathrm{e}-03$ & 3.2788 & $2.7064 \mathrm{e}-03$ & 3.0805 \\
\hline & 256 & $1.0645 \mathrm{e}-03$ & 3.2838 & $1.7919 \mathrm{e}-03$ & 3.0878 \\
\hline & 324 & $7.2300 \mathrm{e}-04$ & 3.2841 & $1.2450 \mathrm{e}-03$ & 3.0916 \\
\hline & 400 & $5.1164 \mathrm{e}-04$ & 3.2819 & $8.9873 \mathrm{e}-04$ & 3.0935 \\
\hline \multirow{9}{*}{$k=3$} & 16 & $6.1615 \mathrm{e}-03$ & NA & $1.1106 \mathrm{e}-02$ & NA \\
\hline & 36 & $8.5780 \mathrm{e}-04$ & 4.8628 & $1.8783 \mathrm{e}-03$ & 4.3829 \\
\hline & 64 & $2.3538 \mathrm{e}-04$ & 4.4952 & $5.5323 \mathrm{e}-04$ & 4.2490 \\
\hline & 100 & $9.1811 \mathrm{e}-05$ & 4.2190 & $2.1883 \mathrm{e}-04$ & 4.1565 \\
\hline & 144 & $4.3638 \mathrm{e}-05$ & 4.0796 & $1.0358 \mathrm{e}-04$ & 4.1021 \\
\hline & 196 & $2.3498 \mathrm{e}-05$ & 4.0158 & $5.5314 \mathrm{e}-05$ & 4.0698 \\
\hline & 256 & $1.3797 \mathrm{e}-05$ & 3.9874 & $3.2210 \mathrm{e}-05$ & 4.0496 \\
\hline & 324 & $8.6387 \mathrm{e}-06$ & 3.9751 & $2.0022 \mathrm{e}-05$ & 4.0366 \\
\hline & 400 & $5.6856 \mathrm{e}-06$ & 3.9704 & $1.3098 \mathrm{e}-05$ & 4.0277 \\
\hline \multirow{9}{*}{$k=4$} & 16 & $6.3759 \mathrm{e}-04$ & NA & $8.0053 \mathrm{e}-04$ & NA \\
\hline & 36 & $8.4088 \mathrm{e}-05$ & 4.9963 & $1.1493 \mathrm{e}-04$ & 4.7869 \\
\hline & 64 & $1.8723 \mathrm{e}-05$ & 5.2215 & $2.7300 \mathrm{e}-05$ & 4.9968 \\
\hline & 100 & $5.7411 \mathrm{e}-06$ & 5.2974 & $8.8037 \mathrm{e}-06$ & 5.0716 \\
\hline & 144 & $2.1728 \mathrm{e}-06$ & 5.3294 & $3.4706 \mathrm{e}-06$ & 5.1055 \\
\hline & 196 & $9.5340 \mathrm{e}-07$ & 5.3436 & $1.5756 \mathrm{e}-06$ & 5.1229 \\
\hline & 256 & $4.6672 \mathrm{e}-07$ & 5.3494 & $7.9397 \mathrm{e}-07$ & 5.1325 \\
\hline & 324 & $2.4852 \mathrm{e}-07$ & 5.3507 & $4.3350 \mathrm{e}-07$ & 5.1379 \\
\hline & 400 & $1.4144 \mathrm{e}-07$ & 5.3494 & $2.5221 \mathrm{e}-07$ & 5.1409 \\
\hline
\end{tabular}

Table 3.1

$L^{2}$ errors for the md-LDG method on uniform meshes having $N$ elements and $Q_{k}$ with $\mathscr{P}^{+} g_{D}$ in (2.16) and $g_{D}$ in (2.15).

[6] P. Castillo, B. Cockburn, I. Perugia, D. Schötzau, An a priori error analysis of the local discontinuous Galerkin method for elliptic problems, SIAM J. Numer. Anal. 38 (2000) 1676-1706.

URL http://dx.doi.org/10.1137/S0036142900371003

[7] P. Castillo, B. Cockburn, D. Schötzau, C. Schwab, Optimal a priori error 


\begin{tabular}{|c|c|c|c|c|c|}
\hline$k$ & $N$ & $\left\|u-u_{N}\right\|_{0, \Omega}$ & Order & $\left\|q_{1}-q_{1, N}\right\|_{0, \Omega}$ & Order \\
\hline \multirow{9}{*}{$k=1$} & 16 & $4.6592 \mathrm{e}-01$ & NA & $8.1159 \mathrm{e}-01$ & NA \\
\hline & 36 & $1.5476 \mathrm{e}-01$ & 2.7182 & $3.5707 \mathrm{e}-01$ & 2.0250 \\
\hline & 64 & $7.6531 \mathrm{e}-02$ & 2.4478 & $2.0960 \mathrm{e}-01$ & 1.8518 \\
\hline & 100 & $4.6716 \mathrm{e}-02$ & 2.2121 & $1.4160 \mathrm{e}-01$ & 1.7575 \\
\hline & 144 & $3.1965 \mathrm{e}-02$ & 2.0811 & $1.0373 \mathrm{e}-01$ & 1.7072 \\
\hline & 196 & $2.3425 \mathrm{e}-02$ & 2.0165 & $8.0097 \mathrm{e}-02$ & 1.6771 \\
\hline & 256 & $1.7969 \mathrm{e}-02$ & 1.9858 & $6.4200 \mathrm{e}-02$ & 1.6568 \\
\hline & 324 & $1.4245 \mathrm{e}-02$ & 1.9716 & $5.2911 \mathrm{e}-02$ & 1.6419 \\
\hline & 400 & $1.1581 \mathrm{e}-02$ & 1.9656 & $4.4560 \mathrm{e}-02$ & 1.6303 \\
\hline \multirow{9}{*}{$k=2$} & 16 & $7.9648 \mathrm{e}-02$ & NA & $1.3297 \mathrm{e}-01$ & NA \\
\hline & 36 & $2.4826 \mathrm{e}-02$ & 2.8750 & $4.4042 \mathrm{e}-02$ & 2.7252 \\
\hline & 64 & $1.0088 \mathrm{e}-02$ & 3.1302 & $1.9528 \mathrm{e}-02$ & 2.8271 \\
\hline & 100 & $4.9201 \mathrm{e}-03$ & 3.2180 & $1.0381 \mathrm{e}-02$ & 2.8317 \\
\hline & 144 & $2.7179 \mathrm{e}-03$ & 3.2550 & $6.2156 \mathrm{e}-03$ & 2.8131 \\
\hline & 196 & $1.6414 \mathrm{e}-03$ & 3.2714 & $4.0433 \mathrm{e}-03$ & 2.7895 \\
\hline & 256 & $1.0596 \mathrm{e}-03$ & 3.2780 & $2.7946 \mathrm{e}-03$ & 2.7661 \\
\hline & 324 & $7.2008 \mathrm{e}-04$ & 3.2794 & $2.0227 \mathrm{e}-03$ & 2.7446 \\
\hline & 400 & $5.0979 \mathrm{e}-04$ & 3.2779 & $1.5178 \mathrm{e}-03$ & 2.7254 \\
\hline \multirow{9}{*}{$k=3$} & 16 & $6.3200 \mathrm{e}-03$ & NA & $1.2346 \mathrm{e}-02$ & NA \\
\hline & 36 & $8.7191 \mathrm{e}-04$ & 4.8852 & $2.4734 \mathrm{e}-03$ & 3.9652 \\
\hline & 64 & $2.3655 \mathrm{e}-04$ & 4.5346 & $8.4950 \mathrm{e}-04$ & 3.7149 \\
\hline & 100 & $9.1681 \mathrm{e}-05$ & 4.2477 & $3.7923 \mathrm{e}-04$ & 3.6142 \\
\hline & 144 & $4.3454 \mathrm{e}-05$ & 4.0951 & $1.9772 \mathrm{e}-04$ & 3.5722 \\
\hline & 196 & $2.3373 \mathrm{e}-05$ & 4.0228 & $1.1436 \mathrm{e}-04$ & 3.5521 \\
\hline & 256 & $1.3720 \mathrm{e}-05$ & 3.9895 & $7.1269 \mathrm{e}-05$ & 3.5411 \\
\hline & 324 & $8.5911 \mathrm{e}-06$ & 3.9745 & $4.7002 \mathrm{e}-05$ & 3.5343 \\
\hline & 400 & $5.6554 \mathrm{e}-06$ & 3.9683 & $3.2404 \mathrm{e}-05$ & 3.5298 \\
\hline \multirow{9}{*}{$k=4$} & 16 & $6.2182 \mathrm{e}-04$ & NA & $1.1241 \mathrm{e}-03$ & NA \\
\hline & 36 & $8.3110 \mathrm{e}-05$ & 4.9634 & $1.7174 \mathrm{e}-04$ & 4.6337 \\
\hline & 64 & $1.8585 \mathrm{e}-05$ & 5.2065 & $4.4120 \mathrm{e}-05$ & 4.7242 \\
\hline & 100 & $5.7104 \mathrm{e}-06$ & 5.2883 & $1.5392 \mathrm{e}-05$ & 4.7191 \\
\hline & 144 & $2.1636 \mathrm{e}-06$ & 5.3231 & $6.5369 \mathrm{e}-06$ & 4.6972 \\
\hline & 196 & $9.5007 \mathrm{e}-07$ & 5.3389 & $3.1802 \mathrm{e}-06$ & 4.6742 \\
\hline & 256 & $4.6532 \mathrm{e}-07$ & 5.3457 & $1.7083 \mathrm{e}-06$ & 4.6538 \\
\hline & 324 & $2.4786 \mathrm{e}-07$ & 5.3476 & $9.8947 \mathrm{e}-07$ & 4.6364 \\
\hline & 400 & $1.4111 \mathrm{e}-07$ & 5.3468 & $6.0803 \mathrm{e}-07$ & 4.6218 \\
\hline
\end{tabular}

Table 3.2

$L^{2}$ errors for the md-LDG method on uniform meshes having $N$ elements and $Q_{k}$ with $g_{D}$ in $(2.16)$ and $\mathscr{P}^{+} g_{D}$ in (2.15).

estimates for the $h p$-version of the local discontinuous Galerkin method for convection-diffusion problems, Math. Comp. 71 (2002) 455-478.

URL http://dx.doi.org/10.1090/S0025-5718-01-01317-5

[8] B. Cockburn, B. Dong, An analysis of the minimal dissipation local discontinuous Galerkin method for convection-diffusion problems, J. Sci. 


\begin{tabular}{|c|c|c|c|c|c|}
\hline$k$ & $N$ & $\left\|u-u_{N}\right\|_{0, \Omega}$ & Order & $\left\|q_{1}-q_{1, N}\right\|_{0, \Omega}$ & Order \\
\hline \multirow{9}{*}{$k=1$} & 16 & $4.4073 \mathrm{e}-01$ & NA & $8.3145 \mathrm{e}-01$ & NA \\
\hline & 36 & $1.4997 \mathrm{e}-01$ & 2.6586 & $3.3132 \mathrm{e}-01$ & 2.2692 \\
\hline & 64 & $7.6020 \mathrm{e}-02$ & 2.3619 & $1.7715 \mathrm{e}-01$ & 2.1764 \\
\hline & 100 & $4.6956 \mathrm{e}-02$ & 2.1591 & $1.1061 \mathrm{e}-01$ & 2.1104 \\
\hline & 144 & $3.2275 \mathrm{e}-02$ & 2.0563 & $7.5814 \mathrm{e}-02$ & 2.0720 \\
\hline & 196 & $2.3684 \mathrm{e}-02$ & 2.0078 & $5.5280 \mathrm{e}-02$ & 2.0491 \\
\hline & 256 & $1.8168 \mathrm{e}-02$ & 1.9854 & $4.2128 \mathrm{e}-02$ & 2.0348 \\
\hline & 324 & $1.4397 \mathrm{e}-02$ & 1.9755 & $3.3187 \mathrm{e}-02$ & 2.0255 \\
\hline & 400 & $1.1696 \mathrm{e}-02$ & 1.9716 & $2.6827 \mathrm{e}-02$ & 2.0191 \\
\hline \multirow{9}{*}{$k=2$} & 16 & $8.7706 \mathrm{e}-02$ & NA & $1.2221 \mathrm{e}-01$ & NA \\
\hline & 36 & $2.6002 \mathrm{e}-02$ & 2.9985 & $3.7367 \mathrm{e}-02$ & 2.9225 \\
\hline & 64 & $1.0340 \mathrm{e}-02$ & 3.2056 & $1.5457 \mathrm{e}-02$ & 3.0685 \\
\hline & 100 & $4.9957 \mathrm{e}-03$ & 3.2599 & $7.7479 \mathrm{e}-03$ & 3.0949 \\
\hline & 144 & $2.7469 \mathrm{e}-03$ & 3.2804 & $4.4011 \mathrm{e}-03$ & 3.1021 \\
\hline & 196 & $1.6547 \mathrm{e}-03$ & 3.2882 & $2.7274 \mathrm{e}-03$ & 3.1041 \\
\hline & 256 & $1.0664 \mathrm{e}-03$ & 3.2899 & $1.8018 \mathrm{e}-03$ & 3.1043 \\
\hline & 324 & $7.2396 \mathrm{e}-04$ & 3.2883 & $1.2501 \mathrm{e}-03$ & 3.1037 \\
\hline & 400 & $5.1216 \mathrm{e}-04$ & 3.2849 & $9.0155 \mathrm{e}-04$ & 3.1026 \\
\hline \multirow{9}{*}{$k=3$} & 16 & $6.2541 \mathrm{e}-03$ & NA & $1.1422 \mathrm{e}-02$ & NA \\
\hline & 36 & $8.6330 \mathrm{e}-04$ & 4.8838 & $1.8950 \mathrm{e}-03$ & 4.4304 \\
\hline & 64 & $2.3623 \mathrm{e}-04$ & 4.5048 & $5.5624 \mathrm{e}-04$ & 4.2608 \\
\hline & 100 & $9.2042 \mathrm{e}-05$ & 4.2241 & $2.1978 \mathrm{e}-04$ & 4.1614 \\
\hline & 144 & $4.3719 \mathrm{e}-05$ & 4.0833 & $1.0395 \mathrm{e}-04$ & 4.1066 \\
\hline & 196 & $2.3530 \mathrm{e}-05$ & 4.0188 & $5.5471 \mathrm{e}-05$ & 4.0741 \\
\hline & 256 & $1.3812 \mathrm{e}-05$ & 3.9897 & $3.2284 \mathrm{e}-05$ & 4.0537 \\
\hline & 324 & $8.6460 \mathrm{e}-06$ & 3.9770 & $2.0060 \mathrm{e}-05$ & 4.0402 \\
\hline & 400 & $5.6895 \mathrm{e}-06$ & 3.9719 & $1.3118 \mathrm{e}-05$ & 4.0309 \\
\hline \multirow{9}{*}{$k=4$} & 16 & $7.0512 \mathrm{e}-04$ & NA & $9.6592 \mathrm{e}-04$ & NA \\
\hline & 36 & $8.8496 \mathrm{e}-05$ & 5.1186 & $1.2736 \mathrm{e}-04$ & 4.9969 \\
\hline & 64 & $1.9184 \mathrm{e}-05$ & 5.3145 & $2.8846 \mathrm{e}-05$ & 5.1621 \\
\hline & 100 & $5.8154 \mathrm{e}-06$ & 5.3489 & $9.0935 \mathrm{e}-06$ & 5.1735 \\
\hline & 144 & $2.1891 \mathrm{e}-06$ & 5.3588 & $3.5430 \mathrm{e}-06$ & 5.1699 \\
\hline & 196 & $9.5789 \mathrm{e}-07$ & 5.3616 & $1.5978 \mathrm{e}-06$ & 5.1659 \\
\hline & 256 & $4.6819 \mathrm{e}-07$ & 5.3610 & $8.0194 \mathrm{e}-07$ & 5.1626 \\
\hline & 324 & $2.4907 \mathrm{e}-07$ & 5.3586 & $4.3672 \mathrm{e}-07$ & 5.1599 \\
\hline & 400 & $1.4167 \mathrm{e}-07$ & 5.3550 & $2.5364 \mathrm{e}-07$ & 5.1575 \\
\hline
\end{tabular}

Table 3.3

$L^{2}$ errors for LDG method on uniform meshes having $N$ elements and $Q_{k}$ with $C_{11}=1$ on edges in $\mathscr{E}_{D}^{+}$and $C_{11}=h$ elsewhere and using the projection $\mathscr{P}^{+} g_{D}$ in (2.16) and (2.15).

Comput. 32 (2007) 233-262.

[9] B. Cockburn, G. Kanschat, I. Perugia, D. Schötzau, Superconvergence of the local discontinuous Galerkin method for elliptic problems on Cartesian grids, 


\begin{tabular}{|c|c|c|c|c|c|}
\hline$k$ & $N$ & $\left\|u-u_{N}\right\|_{0, \Omega}$ & Order & $\left\|q_{1}-q_{1, N}\right\|_{0, \Omega}$ & Order \\
\hline \multirow{9}{*}{$k=1$} & 16 & $4.4408 \mathrm{e}-01$ & NA & $8.9148 \mathrm{e}-01$ & NA \\
\hline & 36 & $1.5330 \mathrm{e}-01$ & 2.6232 & $3.3464 \mathrm{e}-01$ & 2.4166 \\
\hline & 64 & $8.0870 \mathrm{e}-02$ & 2.2231 & $1.8162 \mathrm{e}-01$ & 2.1242 \\
\hline & 100 & $4.9246 \mathrm{e}-02$ & 2.2229 & $1.1413 \mathrm{e}-01$ & 2.0819 \\
\hline & 144 & $3.3047 \mathrm{e}-02$ & 2.1877 & $7.8337 \mathrm{e}-02$ & 2.0642 \\
\hline & 196 & $2.3915 \mathrm{e}-02$ & 2.0983 & $5.7179 \mathrm{e}-02$ & 2.0424 \\
\hline & 256 & $1.8232 \mathrm{e}-02$ & 2.0319 & $4.3607 \mathrm{e}-02$ & 2.0293 \\
\hline & 324 & $1.4413 \mathrm{e}-02$ & 1.9954 & $3.4359 \mathrm{e}-02$ & 2.0236 \\
\hline & 400 & $1.1702 \mathrm{e}-02$ & 1.9781 & $2.7767 \mathrm{e}-02$ & 2.0217 \\
\hline \multirow{9}{*}{$k=2$} & 16 & $8.9470 \mathrm{e}-02$ & NA & $1.3378 \mathrm{e}-01$ & NA \\
\hline & 36 & $2.6581 \mathrm{e}-02$ & 2.9934 & $3.8551 \mathrm{e}-02$ & 3.0686 \\
\hline & 64 & $1.0327 \mathrm{e}-02$ & 3.2865 & $1.5810 \mathrm{e}-02$ & 3.0983 \\
\hline & 100 & $4.9811 \mathrm{e}-03$ & 3.2673 & 7.9147e-03 & 3.1008 \\
\hline & 144 & $2.7521 \mathrm{e}-03$ & 3.2613 & $4.4963 \mathrm{e}-03$ & 3.1011 \\
\hline & 196 & $1.6626 \mathrm{e}-03$ & 3.2694 & $2.7811 \mathrm{e}-03$ & 3.1166 \\
\hline & 256 & $1.0725 \mathrm{e}-03$ & 3.2830 & $1.8320 \mathrm{e}-03$ & 3.1263 \\
\hline & 324 & $7.2803 \mathrm{e}-04$ & 3.2893 & $1.2672 \mathrm{e}-03$ & 3.1293 \\
\hline & 400 & $5.1475 \mathrm{e}-04$ & 3.2901 & $9.1140 \mathrm{e}-04$ & 3.1281 \\
\hline \multirow{9}{*}{$k=3$} & 16 & $6.3133 \mathrm{e}-03$ & NA & $1.2287 \mathrm{e}-02$ & NA \\
\hline & 36 & $8.8244 \mathrm{e}-04$ & 4.8530 & $1.9812 \mathrm{e}-03$ & 4.5005 \\
\hline & 64 & $2.6889 \mathrm{e}-04$ & 4.1309 & $5.9925 \mathrm{e}-04$ & 4.1566 \\
\hline & 100 & $1.0247 \mathrm{e}-04$ & 4.3233 & $2.3454 \mathrm{e}-04$ & 4.2039 \\
\hline & 144 & $4.6281 \mathrm{e}-05$ & 4.3597 & $1.0934 \mathrm{e}-04$ & 4.1857 \\
\hline & 196 & $2.4122 \mathrm{e}-05$ & 4.2270 & $5.7944 \mathrm{e}-05$ & 4.1193 \\
\hline & 256 & $1.3945 \mathrm{e}-05$ & 4.1040 & $3.3657 \mathrm{e}-05$ & 4.0685 \\
\hline & 324 & $8.6757 \mathrm{e}-06$ & 4.0295 & $2.0913 \mathrm{e}-05$ & 4.0400 \\
\hline & 400 & $5.6972 \mathrm{e}-06$ & 3.9916 & $1.3683 \mathrm{e}-05$ & 4.0264 \\
\hline \multirow{9}{*}{$k=4$} & 16 & $7.0512 \mathrm{e}-04$ & NA & $9.6592 \mathrm{e}-04$ & NA \\
\hline & 36 & $8.8496 \mathrm{e}-05$ & 5.1186 & $1.2736 \mathrm{e}-04$ & 4.9969 \\
\hline & 64 & $1.9184 \mathrm{e}-05$ & 5.3145 & $2.8846 \mathrm{e}-05$ & 5.1621 \\
\hline & 100 & $5.7722 \mathrm{e}-06$ & 5.3548 & $9.3721 \mathrm{e}-06$ & 5.1318 \\
\hline & 144 & $2.1891 \mathrm{e}-06$ & 5.3031 & $3.5430 \mathrm{e}-06$ & 5.1248 \\
\hline & 196 & $9.5789 \mathrm{e}-07$ & 5.3616 & $1.5978 \mathrm{e}-06$ & 5.1659 \\
\hline & 256 & $4.6819 \mathrm{e}-07$ & 5.3610 & $8.0194 \mathrm{e}-07$ & 5.1626 \\
\hline & 324 & $2.4907 \mathrm{e}-07$ & 5.3586 & $4.3672 \mathrm{e}-07$ & 5.1599 \\
\hline & 400 & $1.4167 \mathrm{e}-07$ & 5.3550 & $2.5364 \mathrm{e}-07$ & 5.1575 \\
\hline
\end{tabular}

Table 3.4

$L^{2}$ errors for the LDG method on uniform meshes having $N$ elements and $Q_{k}$ with $C_{11}=1$ on edges in $\mathscr{E}_{D}^{+} \cup \mathscr{E}_{S}, C_{11}=0$ elsewhere and using the projection $\mathscr{P}^{+} g_{D}$ in (2.16) and (2.15).

SIAM J. Numer. Anal. 39 (2001) 264-285.

[10] B. Cockburn, G. Karniadakis, C.-W. Shu, Discontinuous Galerkin Methods: Theory, Computation and Applications, vol. 11 of Lecture Notes in 


\begin{tabular}{|c|c|c|c|c|c|}
\hline$k$ & $N$ & $\left\|u-u_{N}\right\|_{0, \Omega}$ & Order & $\left\|q_{1}-q_{1, N}\right\|_{0, \Omega}$ & Order \\
\hline \multirow{9}{*}{$k=1$} & 16 & $4.4571 \mathrm{e}-01$ & NA & $8.8583 \mathrm{e}-01$ & NA \\
\hline & 36 & $1.5242 \mathrm{e}-01$ & 2.6464 & $3.5325 \mathrm{e}-01$ & 2.2674 \\
\hline & 64 & $7.8217 \mathrm{e}-02$ & 2.3191 & $1.9103 \mathrm{e}-01$ & 2.1368 \\
\hline & 100 & $4.8570 \mathrm{e}-02$ & 2.1353 & $1.2092 \mathrm{e}-01$ & 2.0495 \\
\hline & 144 & $3.3389 \mathrm{e}-02$ & 2.0556 & $8.3758 \mathrm{e}-02$ & 2.0139 \\
\hline & 196 & $2.4453 \mathrm{e}-02$ & 2.0207 & $6.1531 \mathrm{e}-02$ & 2.0006 \\
\hline & 256 & $1.8710 \mathrm{e}-02$ & 2.0047 & $4.7134 \mathrm{e}-02$ & 1.9961 \\
\hline & 324 & $1.4788 \mathrm{e}-02$ & 1.9973 & $3.7263 \mathrm{e}-02$ & 1.9952 \\
\hline & 400 & $1.1986 \mathrm{e}-02$ & 1.9939 & $3.0196 \mathrm{e}-02$ & 1.9957 \\
\hline \multirow{9}{*}{$k=2$} & 16 & $1.0181 \mathrm{e}-01$ & NA & $1.5434 \mathrm{e}-01$ & NA \\
\hline & 36 & $3.1291 \mathrm{e}-02$ & 2.9098 & $5.1499 \mathrm{e}-02$ & 2.7070 \\
\hline & 64 & $1.2166 \mathrm{e}-02$ & 3.2836 & $2.1387 \mathrm{e}-02$ & 3.0547 \\
\hline & 100 & $5.7263 \mathrm{e}-03$ & 3.3772 & $1.0580 \mathrm{e}-02$ & 3.1539 \\
\hline & 144 & $3.0811 \mathrm{e}-03$ & 3.3994 & $5.9172 \mathrm{e}-03$ & 3.1873 \\
\hline & 196 & $1.8246 \mathrm{e}-03$ & 3.3989 & $3.6140 \mathrm{e}-03$ & 3.1986 \\
\hline & 256 & $1.1602 \mathrm{e}-03$ & 3.3904 & $2.3569 \mathrm{e}-03$ & 3.2012 \\
\hline & 324 & $7.7929 \mathrm{e}-04$ & 3.3791 & $1.6168 \mathrm{e}-03$ & 3.2000 \\
\hline & 400 & $5.4655 \mathrm{e}-04$ & 3.3671 & $1.1544 \mathrm{e}-03$ & 3.1972 \\
\hline \multirow{9}{*}{$k=3$} & 16 & $6.5114 \mathrm{e}-03$ & NA & $1.2343 \mathrm{e}-02$ & NA \\
\hline & 36 & $8.9963 \mathrm{e}-04$ & 4.8816 & $2.0119 \mathrm{e}-03$ & 4.4739 \\
\hline & 64 & $2.4500 \mathrm{e}-04$ & 4.5213 & $5.9422 \mathrm{e}-04$ & 4.2394 \\
\hline & 100 & $9.5249 \mathrm{e}-05$ & 4.2339 & $2.3911 \mathrm{e}-04$ & 4.0796 \\
\hline & 144 & $4.5131 \mathrm{e}-05$ & 4.0968 & $1.1492 \mathrm{e}-04$ & 4.0184 \\
\hline & 196 & $2.4221 \mathrm{e}-05$ & 4.0371 & $6.2043 \mathrm{e}-05$ & 3.9989 \\
\hline & 256 & $1.4177 \mathrm{e}-05$ & 4.0109 & $3.6395 \mathrm{e}-05$ & 3.9945 \\
\hline & 324 & $8.8517 \mathrm{e}-06$ & 3.9992 & $2.2733 \mathrm{e}-05$ & 3.9955 \\
\hline & 400 & $5.8112 \mathrm{e}-06$ & 3.9941 & $1.4918 \mathrm{e}-05$ & 3.9981 \\
\hline \multirow{9}{*}{$k=4$} & 16 & $8.7887 \mathrm{e}-04$ & NA & $1.3507 \mathrm{e}-03$ & NA \\
\hline & 36 & $1.1802 \mathrm{e}-04$ & 4.9517 & $2.0218 \mathrm{e}-04$ & 4.6840 \\
\hline & 64 & $2.5051 \mathrm{e}-05$ & 5.3878 & $4.6586 \mathrm{e}-05$ & 5.1024 \\
\hline & 100 & $7.3412 \mathrm{e}-06$ & 5.5006 & $1.4528 \mathrm{e}-05$ & 5.2219 \\
\hline & 144 & $2.6792 \mathrm{e}-06$ & 5.5285 & $5.5639 \mathrm{e}-06$ & 5.2641 \\
\hline & 196 & $1.1425 \mathrm{e}-06$ & 5.5289 & $2.4655 \mathrm{e}-06$ & 5.2800 \\
\hline & 256 & $5.4675 \mathrm{e}-07$ & 5.5193 & $1.2173 \mathrm{e}-06$ & 5.2854 \\
\hline & 324 & $2.8585 \mathrm{e}-07$ & 5.5062 & $6.5313 \mathrm{e}-07$ & 5.2860 \\
\hline & 400 & $1.6026 \mathrm{e}-07$ & 5.4920 & $3.7428 \mathrm{e}-07$ & 5.2843 \\
\hline
\end{tabular}

Table 3.5

$L^{2}$ errors for the LDG method on uniform meshes having $N$ elements and $Q_{k}$ with $C_{11}=1$ on all edges and using the projection $\mathscr{P}^{+} g_{D}$ in (2.16) and (2.15).

Computational Science and Engineering, Springer, Berlin, 2000.

[11] B. Cockburn, C.-W. Shu, TVB Runge-Kutta local projection discontinuous Galerkin methods for scalar conservation laws II: General framework, Math. Comp. 52 (1989) 411-435.

URL http://dx.doi.org/10.2307/2008474 
[12] B. Cockburn, C.-W. Shu, The local discontinuous Galerkin finite element method for time dependent convection-diffusion systems, SIAM J. Numer. Anal. 35 (1998) 2240-2463. URL http://dx.doi.org/10.1007/s10915-007-9130-3

[13] B. Cockburn, C.-W. Shu, The Runge-Kutta discontinuous Galerkin finite element method for conservation laws V: Multidimensional systems, J. Comput. Phys. 141 (1998) 199-224.

URL http://dx.doi.org/10.1006/jcph.1998.5892

[14] B. Cockburn, C.-W. Shu, Foreword for the special issue on discontinuous Galerkin methods, J. Sci. Comput. 22/23 (2005) 1-2. URL http://dx.doi.org/10.1007/s10915-004-4131-y

[15] B. Cockburn, C.-W. Shu, Foreword, proceedings of the first international symposium on dg methods, J. Sci. Comput. 40 (2009) 1-3. URL http://dx.doi.org/10.1007/s10915-009-9298-9

[16] C. Dawson, Foreword for the special issue on discontinuous Galerkin methods, Comput. Methods Appl. Mech. Engrg. 195 (2006) 3183-3183.

URL http://dx.doi.org/10.1016/j.cma.2005.06.010

[17] W. Reed, T. Hill, Triangular mesh methods for the neutron transport equation, Tech. Rep. LA-UR-73-479, Los Alamos Scientific Laboratory, Los Alamos (1973). 